\title{
The policy art of the "trade-off" for combatting COVID-19
}

\section{Jong-Koo Lee}

Department of Family Medicine, Seoul National University College of Medicine, Seoul, Korea

Received: June 10, 2021

Accepted: June 14, 2021

Corresponding author: Jong-Koo Lee Department of Family Medicine, Seoul National University College of Medicine, 103 Daehak-ro, Jongno-gu, Seoul 03080, Korea E-mail: docmohw@snu.ac.kr
In light of the recently held G7 Health Ministers Meeting, expectations have grown for the G7 summit meeting that will be held in June 2021 in the United Kingdom. At a highly apt time, in a recent speech, the World Health Organization (WHO) director-general Tedros Adhanom Ghebreyesus emphasized the need to move away from vaccine nationalism and inequity in order to escape from the coronavirus disease 2019 (COVID-19) pandemic, and discussed the need for a new pandemic treaty [1]. A treaty would foster improved sharing, trust, and accountability, and help to strengthen national, regional, and global capacities for global health security. Despite the declaration of an international health crisis with the early recognition of COVID-19 as a public health emergency of international concern (PHEIC), and the emphasis on solidarity and alliance, nearly all countries and jurisdictions have violated the IHR temporary recommendations advised by WHO COVID-19 IHR Committee. Although President Biden has suggested that 500 million doses of the Moderna vaccine will be donated to save lives in lowerincome countries [2], I wonder how advanced countries leading the international community will overcome this crisis, and how our health diplomats will share our achievements with other countries.

Even though the strong suppression policy involving non-pharmaceutical interventions was effective in the first half of the COVID-19 pandemic, the outcome of the battle of the second half depends on achieving herd immunity through effective vaccines. We have succeeded in quarantine by rapid testing, contact tracing, and prevention of hospital overload by implementing designated COVID-19 treatment facilities, but to reach herd immunity in 6 months seems challenging. In Israel, the United Kingdom, and the United States, where vaccination has been accelerated, the prevalence and mortality rates of COVID-19 are significantly decreasing. However, it is difficult to achieve full herd immunity and eliminate COVID-19, because regulatory authorities allowed the emergency use of vaccines and did not recommend for use in those lesser than 12 years of age (Pfizer-BioNThech) or 18 years of age (Moderna, Janssen) [3] due to a lack of complete efficacy data. Anyway recent studies on the efficacy and the safety of the BNT162b2 COVID-19 vaccine in adolescents will facilitate the achievement of herd immunity [4]. But, outbreaks and reports of severe cases are increasing, in addition, the emergence of variants of COVID-19 and the decline of antibody titers after vaccination has suggested the need for booster vaccination, and public health authorities in some countries are concerned about the increase in patients after the government hastily lifted guidelines for social distancing and mask-wearing. In the Republic of Korea, early 
vaccine acquisition and rollout were delayed, and vaccine hesitancy due to controversy regarding the rare event of cerebral venous sinus thrombosis linked the AstraZeneca vaccine made an impact on the race towards vaccination. The one-dose use of the Janssen vaccine targeting veterans is also scheduled to start after approval by the regulatory authorities, which will lead to further progress in this race. This provides an optimistic perspective that 14 million people will be able to receive the shot. However, more active persuasion is needed. Infectious diseases have a large external effect, and in order to protect the entire society, herd immunity is required to block transmission. A sophisticated explanatory model is needed for political decision-making and persuasion of the public. Non-pharmacological intervention policies, vaccine rollout, and border opening are inextricably linked with the COVID-19 infection rate and economic losses. Therefore, modeling to determine which policy option to choose and at which time to implement it becomes important, and simulation of the options would help in decision-making. Although the government has never clearly stated whether its policy goal regarding COVID-19 is to eliminate the disease or to mitigate its consequences, the trade-off between different options is the art of decisionmaking for the elimination of COVID-19. Public health measures, economic losses, democratic solidarity, and civil liberties are important factors when evaluating pandemic responses. The Republic of Korea has been recognized as one of the 5 best Organization of Economic Co-operation and Development (OECD) countries in terms of eliminating COVID-19 [5]. The Korean government has aimed to lower $\mathrm{R}(\mathrm{t})$ below 1 and then shift to a mitigation policy once it falls below 1; thus, the Republic of Korea is an example of a country with a tight suppression policy, not a country with an aggressive/moderate elimination policy [6].

Most of all, an authentic attitude on the part of decisionmakers makes it possible to persuade the public to participate in policy uptake. The government's responsibility is to secure a safe vaccine and to make decisions transparently. The responsibility of the media is to provide accurate and detailed reports on the benefits of vaccines according to the science and the evidence. The accountability of the people is to get vaccinated, wear masks, and maintain social distancing.
No one is safe until everyone is safe. When everyone is responsible and accountable in their role, COVID-19 will be eliminated.

\section{Notes}

\section{Ethics Approval}

Not applicable.

\section{Conflicts of Interest}

The author has no conflicts of interest to declare.

\section{Funding}

None.

\section{References}

1. World Health Organization (WHO). WHO Director-General's keynote speech at the St Petersburg International Economic Forum '21 [Internet]. Geneva: WHO; 2021 [cited 2021 Jun 10]. Available from: https://www.who.int/director-general/speeches/detail/who-directorgeneral-s-keynote-speech-at-the-st-petersburg-internationaleconomic-forum-21.

2. Pager T, Rauhala E. Biden administration to buy 500 million Pfizer coronavirus vaccine doses to donate to the world [Internet]. The Washington Post; 2021 Jun 10 [cited 2021 Jun 10]. Available from: https:// www.washingtonpost.com/politics/biden-vaccine-donate/2021/06/09/ c2744674-c934-11eb-93fa-9053a95eb9f2_story.html.

3. COVID-19 Vaccines. The FDA has regulatory processes in place to facilitate the development of COVID-19 vaccines that meet the FDA's rigorous scientific standards [Internet]. Silver Spring, MD: U.S. Food and Drug Administration; 2021 [cited 2021 Jun 10]. Available from: https://www.fda.gov/emergency-preparedness-and-response/ coronavirus-disease-2019-covid-19/covid-19-vaccines.

4. Frenck RW Jr, Klein NP, Kitchin N, et al. Safety, immunogenicity, and efficacy of the BNT162b2 Covid-19 vaccine in adolescents. N Engl J Med 2021 May 27 [Epub]. https://www.nejm.org/doi/10.1056/ NEJMoa2107456.

5. Oliu-Barton M, Pradelski BSR, Aghion P, et al. SARS-CoV-2 elimination, not mitigation, creates best outcomes for health, the economy, and civil liberties. Lancet 2021;397:2234-6.

6. The University of Melbourne. COVID-19 pandemic trade-offs [Internet]. Melbourne: The University of Melbourne; 2021 [cited 2021 Jun 10]. Available from: https://populationinterventions.science. unimelb.edu.au/pandemic-trade-offs/. 